\title{
Higher Inpatient Morbidity and Mortality in Biliary Pancreatitis Compared to Hypertriglyceridemia-Induced Pancreatitis: A Nationwide Retrospective Study
}

\author{
Hafeez Shaka ${ }^{1}$, Jennifer C. Asotibe ${ }^{1}$, Ikechukwu Achebe ${ }^{1}$, Garima Pudasaini ${ }^{1}$ \\ 1. Internal Medicine, John H. Stroger, Jr. Hospital of Cook County, Chicago, USA
}

Corresponding author: Ikechukwu Achebe, ikechukwu.achebe@cookcountyhhs.org

\begin{abstract}
Introduction

Hypertriglyceridemia (HTG)-induced pancreatitis is the third most common cause of acute pancreatitis after gallstone disease and alcohol. We analyzed data from the National (Nationwide) Inpatient Sample (NIS) with the aim of evaluating the outcomes of patients with HTG-induced pancreatitis when compared to those with biliary-induced pancreatitis.
\end{abstract}

\section{Methods}

The NIS database was sourced for data involving adult hospitalizations for HTG-induced pancreatitis in the United States between January 1, 2016 and December 31, 2017. The main outcome was mortality in patients with biliary pancreatitis vs HTG pancreatitis. Secondary outcomes were the incidence of sepsis, septic shock, non-ST-elevation myocardial infarction (NSTEMI), blood transfusion requirements, acute kidney failure, acute respiratory distress syndrome (ARDS), and length of hospital stay.

\section{Results}

A total of 575,230 patients were admitted with a diagnosis of acute pancreatitis, $18.2 \%$ of which were classified as having HTG pancreatitis. The in-hospital mortality for pancreatitis was $0.59 \%$. Patients with HTG pancreatitis had lower odds of in-hospital mortality (adjusted odds ratio [aOR]: 0.74, 95\% CI 0.5820.934, $\mathrm{p}=0.012$ ) compared to those with biliary pancreatitis. Patients with HTG pancreatitis had less odds of developing comorbid sepsis (aOR: 0.52, 95\% CI 0.441-0.612, p<0.001), septic shock (aOR: 0.64, 95\% CI 0.482$0.851, \mathrm{p}<0.001$ ), and NSTEMI (aOR: $0.70,95 \%$ CI $0.535-0.926, \mathrm{p}<0.001$ ) and had less odds of requiring transfusion of blood products (aOR: $0.57,95 \%$ CI $0.478-0.678, \mathrm{p}<0.001$ ) when compared to those with biliary pancreatitis. Patients with HTG pancreatitis also had a lower average length of hospital stay and lower total hospital charges compared to those with biliary pancreatitis. There was no statistical difference, however, in acute kidney failure and ARDS between the two groups.

Received 08/19/2020

Review began 08/21/2020 Review ended 09/06/2020 Published 09/10/2020

๑) Copyright 2020

Shaka et al. This is an open access article distributed under the terms of the Creative Commons Attribution License CC-BY 4.0., which permits unrestricted use, distribution, and reproduction in any medium, provided the original author and source are credited.

\section{Conclusion}

Patients with HTG-induced pancreatitis possibly have better inpatient outcomes including mortality when compared to those with biliary-induced pancreatitis.

Categories: Endocrinology/Diabetes/Metabolism, Internal Medicine, Gastroenterology

Keywords: hypertriglyceridemia, acute pancreatitis, biliary pancreatitis, mortality, gallstone pancreatitis

\section{Introduction}

Hypertriglyceridemia (HTG)-induced pancreatitis is the most common cause of acute pancreatitis after gallstone disease and alcohol abuse [1,2]. The incidence of HTG pancreatitis has been variously reported; however, most recent literature suggests the incidence to range from $7 \%$ to as high as $38 \%$ of patients presenting with acute pancreatitis [2-4]. This proportion increases in pregnancy, with HTG causing nearly $50 \%$ of acute pancreatitis cases in pregnancy $[5,6]$. While some patients inherit genes that predispose to HTG, others acquire the disease as a consequence of their obesity, diabetes, pregnancy, hypothyroidism, and medications [7-12].

Disease pathophysiology of HTG-induced pancreatitis is still being understood; however, accumulation of lipotoxic triglyceride metabolites, specifically free fatty acids, is thought to injure pancreatic tissue and cause subsequent inflammation. While triglyceride levels of above $1,000 \mathrm{mg} / \mathrm{dl}$ are thought to increase the risk of acute pancreatitis, the true level of HTG at which pancreatitis occurs is unknown $[5,13]$. 
Gallstone disease is responsible for up to $70 \%$ of acute pancreatitis cases and surpasses HTG as the leading cause of acute pancreatitis [3-7]. Gallstone obstruction of the ampulla causes restricted outflow of pancreatic secretions and occasionally bile reflux into the pancreatic duct leading to irritation and inflammation of the pancreas. Clinically, the presentation of gallstone pancreatitis is indistinguishable from HTG-induced pancreatitis. Both patients present with severe, epigastric abdominal pain, and associated nausea and vomiting. Demographically, gallstone pancreatitis has been found to be more common in females, while HTG-induced pancreatitis presents earlier than gallstone pancreatitis [14-18].

To date, studies investigating morbidity and mortality outcomes between HTG and gallstone pancreatitis are infrequent and inconclusive. While some studies report increased morbidity, mortality, and pancreatitis severity in patients with HTG pancreatitis compared to gallstone-induced disease, other studies have found no difference between the two groups [1,13,19-22]. To the best of our knowledge, this study represents the first large-scale nationwide study evaluating outcomes of HTG-induced pancreatitis compared to biliaryinduced pancreatitis.

\section{Materials And Methods Study design}

The present study is a retrospective cohort study that included adult admissions for HTG pancreatitis in the United States that occurred between January 2016 and December 2017. Data from the calendar years 2016 to 2017 were extracted from the NIS database and used in this study. The National (Nationwide) Inpatient Sample (NIS) provides data from inpatient hospital admissions that are acquired through billing data submitted to statewide organizations. Currently, the NIS covers over $97 \%$ of the population across 47 U.S. states and the District of Columbia, and has been shown to provide reliable inpatient estimates of comorbidity and disease prevalence [23,24]. International Classification of Diseases (Tenth Revision) (ICD10), and Clinical Modification Procedure Coding System (CM/PCS) were used to conduct this study. The NIS utilizes two diagnosis categories, principal and secondary. The principal diagnosis represents the pathology that caused admission, while secondary diagnosis refers to all other assigned diagnoses [24].

\section{Study population}

NIS data were gathered from years 2016 through 2017 for patients aged 18 years and above who had a principal discharge diagnosis of acute pancreatitis (K85). Since there is no specific ICD-10 code for HTG pancreatitis, we formed this group by combining patients with a principal diagnosis of acute pancreatitis, not due to gallstones, alcohol, or drugs, and matched them with a secondary diagnosis of HTG, including pure hyperglyceridemia (E78.1), mixed hyperlipidemia (E78.2), hyperchylomicronemia (E78.3), other hyperlipidemia (E78.4), hyperlipidemia unspecified (E78.5), and lipoprotein deficiency (E78.6). A comparison group of patients with biliary acute pancreatitis (K85.1) along with patients with either idiopathic or unknown etiology of acute pancreatitis with concomitant cholelithiasis or cholecystitis (K80, K81) was generated to compare outcomes. Patients were excluded if they were younger than 18 years of age, had acute pancreatitis as a secondary diagnosis, or pancreatitis caused by other etiologies [24].

\section{Study outcomes}

The primary outcome was to compare inpatient mortality among patients admitted for HTG vs biliary acute pancreatitis. Secondary outcomes in this population include the development of sepsis, septic shock, nonST segment elevation myocardial infarction (NSTEMI), need for transfusion of blood products, acute kidney failure, acute respiratory distress syndrome (ARDS), as well as mean length of hospitalization and mean total hospital charges.

\section{Statistical analysis}

Data were analyzed using the statistical software Stata ${ }^{\circledR}$ Version 16 (Stata Corp, College Station, TX, USA). All data analyses performed in this study utilized the Healthcare Cost and Utilization Project (HCUP) regulations NIS. Chi-squared testing was employed to compare characteristics between patients with HTG pancreatitis and other etiologies of acute pancreatitis [24]. Multivariate regression analysis was then used to remove the effect of possible confounders, and also to calculate outcomes (primary, and secondary). Univariate screening was utilized to confirm factors that had an effect on outcomes. Variables with a p-value of less than 0.2 , were included in multivariate regression analysis. A p-value of less than 0.05 was our indication of statistical significance.

\section{Ethical considerations}

In an effort to protect patient information, the NIS has removed all possible patient and or hospital identifiers. With patient identifiers removed from data, this study did not require Institutional Review Board approval [24].

\section{Results}




\section{Cureus}

\section{Patient characteristics}

The combined NIS database for 2016 and 2017 contained over 71 million weighted hospital discharges; of which 575,230 have a principal discharge diagnosis of acute pancreatitis [24]. Of these patients, $18.2 \%$ were classified as having HTG pancreatitis, while the other subgroup comprises various other etiologies of acute pancreatitis.

Patients with HTG pancreatitis were significantly older ( 56.7 vs 50.8 years, $\mathrm{p}<0.001$ ), and had a higher proportion of whites and Hispanics. These patients had more comorbidities, including diabetes $(52.6 \% \mathrm{vs}$ $20.6 \%$, $\mathrm{p}<0.001$ ), obesity ( $24.6 \%$ vs $15.2 \%$, $\mathrm{p}<0.001$ ), hypothyroidism ( $13.1 \%$ vs $7.9 \%, \mathrm{p}<0.001)$, chronic ischemic heart disease ( $21.7 \%$ vs $9.1 \%, \mathrm{p}<0.001)$, and hypertension $(60.3 \%$ vs $43.9 \%, \mathrm{p}<0.001)$ compared with patients with other etiologies of acute pancreatitis. Patients with HTG pancreatitis had a lower proportion of prior tobacco use. Patient and hospital characteristics are detailed in Table 1.

\begin{tabular}{|c|c|c|c|}
\hline Variable $(n=575,230)$ & Non-HTG pancreatitis, $n=470,765$ (81.8)\% & HTG pancreatitis, n=104,465 (18.2)\% & P-value \\
\hline \multicolumn{4}{|l|}{ Patient characteristics } \\
\hline Age, mean & 50.8 & 56.7 & $<0.001$ \\
\hline Women & 47.2 & 45.5 & $<0.001$ \\
\hline \multicolumn{2}{|l|}{ Racial distribution } & & $<0.001$ \\
\hline White & 61.6 & 64.4 & \\
\hline Black & 17.3 & 12.9 & \\
\hline Hispanic & 12.0 & 13.3 & \\
\hline Others & 9.1 & 9.4 & \\
\hline \multicolumn{2}{|l|}{ Insurance type } & & $<0.001$ \\
\hline Medicaid & 29.5 & 43.6 & \\
\hline Medicare & 27.1 & 17.4 & \\
\hline Private & 33.3 & 33.3 & \\
\hline Uninsured & 10.1 & 5.7 & \\
\hline \multicolumn{2}{|c|}{ Charison Comorbidity Index score } & & $<0.001$ \\
\hline 0 & 45.1 & 21.6 & \\
\hline 1 & 29.0 & 33.5 & \\
\hline 2 & 12.3 & 20.7 & \\
\hline$\geq 3$ & 13.6 & 24.2 & \\
\hline \multicolumn{2}{|c|}{ Median annual income in patient's zip code, US\$\# } & & 0.033 \\
\hline $1-43,999$ & 33.2 & 32.4 & \\
\hline $44,000-55,999$ & 26.5 & 27.4 & \\
\hline 56,000-73,999 & 23.1 & 23.4 & \\
\hline$\geq 74,000$ & 17.2 & 16.8 & \\
\hline \multicolumn{4}{|l|}{ Comorbidities } \\
\hline Diabetes & 20.6 & 52.6 & $<0.001$ \\
\hline Hypertension & 43.9 & 60.3 & $<0.001$ \\
\hline Smoking history & 48.3 & 45.4 & $<0.001$ \\
\hline $\mathrm{CHF}$ & 5.0 & 8.1 & $<0.001$ \\
\hline CKD & 6.1 & 11.0 & $<0.001$ \\
\hline Hypothyroidism & 7.9 & 13.1 & $<0.001$ \\
\hline
\end{tabular}




\section{Cureus}

\begin{tabular}{|c|c|c|c|}
\hline Obesity & 15.2 & 24.6 & $<0.001$ \\
\hline Chronic IHD & 9.1 & 21.7 & $<0.001$ \\
\hline Prior CVA & 0.7 & 1.2 & $<0.001$ \\
\hline COPD & 9.0 & 12.2 & $<0.001$ \\
\hline Anemia & 17.4 & 17.1 & 0.217 \\
\hline \multicolumn{4}{|c|}{ Hospital characteristics } \\
\hline \multicolumn{2}{|l|}{ Hospital region } & & $<0.001$ \\
\hline Northeast & 17.1 & 15.6 & \\
\hline Midwest & 22.3 & 24.1 & \\
\hline South & 40.5 & 42.6 & \\
\hline West & 20.1 & 17.7 & \\
\hline \multicolumn{2}{|l|}{ Hospital bed size } & & 0.003 \\
\hline Small & 23.6 & 22.4 & \\
\hline Medium & 30.7 & 30.8 & \\
\hline Large & 45.7 & 46.8 & \\
\hline Urban location & 88.1 & 86.8 & $<0.001$ \\
\hline Teaching hospital & 60.2 & 58.9 & 0.002 \\
\hline \multicolumn{4}{|c|}{ Non-HTG vs HTG } \\
\hline \multicolumn{4}{|l|}{ \#: for 2017} \\
\hline \multicolumn{4}{|c|}{$\begin{array}{l}\text { CHF, congestive heart failure; CKD, chronic kidney disease; COPD, chronic obstructive pulmonary disease; CVA, cerebrovascular accident; HTG, } \\
\text { hypertriglyceridemia; IHD:, ischemic heart disease }\end{array}$} \\
\hline
\end{tabular}

\section{Primary outcome: in-hospital mortality}

The in-hospital mortality for patients with acute pancreatitis was $0.59 \%$. Patients with HTG pancreatitis had a lower odds of in-hospital mortality (adjusted odds ratio [aOR]: 0.74, 95\% CI 0.582-0.934, p=0.012) compared to those with biliary pancreatitis when adjusted for comorbidities (age, sex, racial distribution, Charlson disease severity index, hospital location, hypertension, smoking history, chronic kidney disease, congestive heart failure, obesity, diabetes, chronic ischemic heart disease, chronic obstructive pulmonary disease, hypothyroidism, and liver disease) using multivariate logistic regression analysis.

\section{Secondary outcomes}

Patients with HTG pancreatitis had less odds of having comorbid sepsis (aOR: 0.52, 95\% CI 0.441-0.612, $\mathrm{p}<0.001$ ), septic shock (aOR: 0.64, 95\% CI 0.482-0.851, p<0.001), NSTEMI (aOR: 0.70, 95\% CI 0.535-0.926, $\mathrm{p}<0.001$ ), and transfusion of blood products (aOR: $0.57,95 \%$ CI 0.478-0.678, $\mathrm{p}<0.001$ ) compared to those with biliary pancreatitis. Patients with HTG pancreatitis also had a lower mean length of stay and mean total hospital charges compared to those with biliary pancreatitis.

There was no statistical difference in comorbid acute kidney failure and ARDS between the two groups. Detailed outcomes are provided in Table 2. 


\section{Cureus}

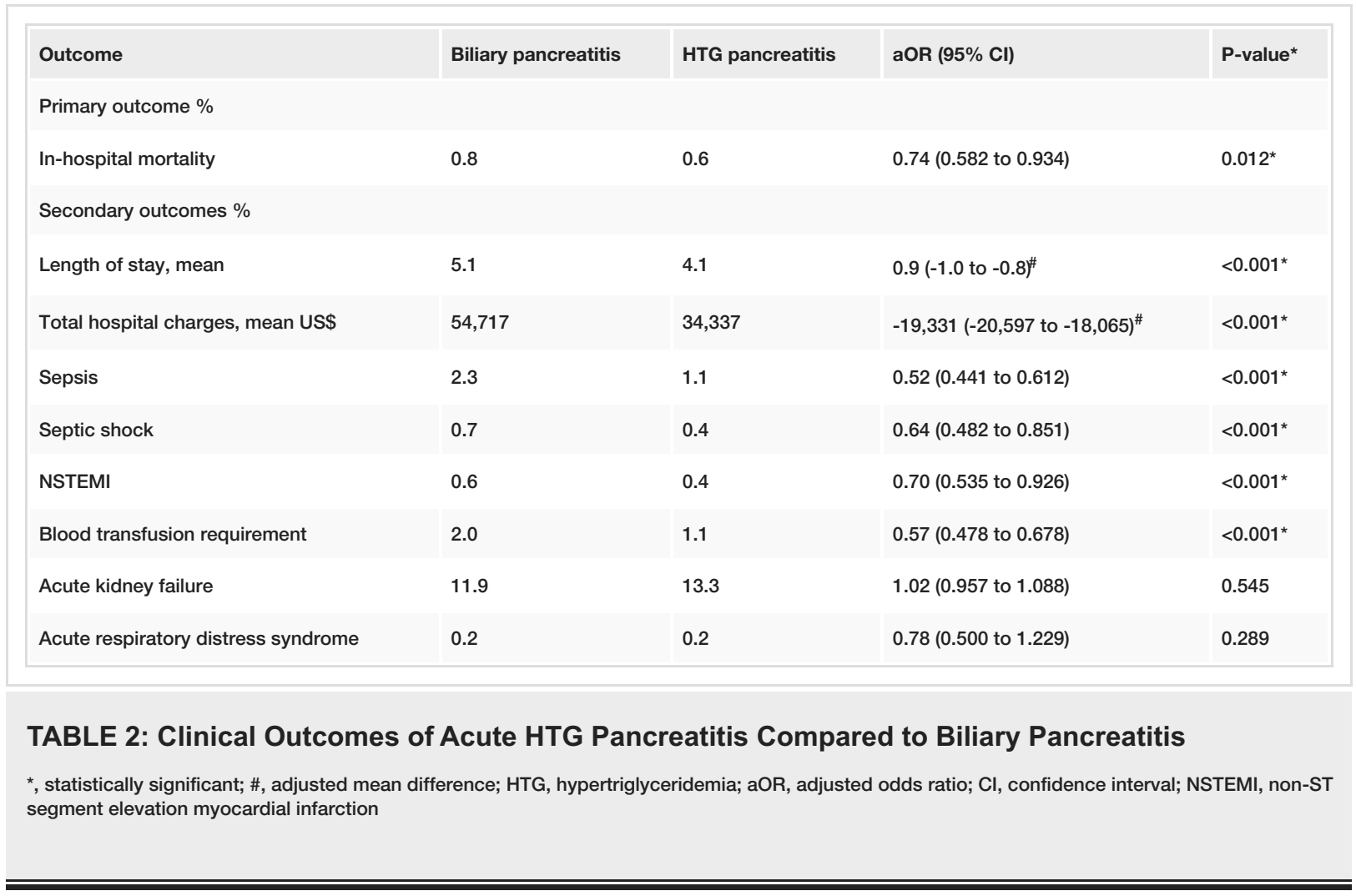

\section{Discussion}

Acute pancreatitis is a recognized cause of increased morbidity and mortality in hospitalized patients and leads as a top cause of gastrointestinal related admissions. With continued increases in population obesity, poor dieting, and gallstone disease the national incidence of acute pancreatitis is expected to rise $[4,5]$. Our study is one of the few large-scale and nationwide cohort studies to evaluate outcomes of HTG-induced pancreatitis compared to biliary-induced pancreatitis.

Compared to other studies like Deng et al., who reported increased incidence of multiorgan failure, shock, renal failure, and overall mortality in patients admitted with HTG, our study found patients with HTG pancreatitis to have lower odds of in-hospital mortality compared to those with biliary pancreatitis (aOR: $0.74, p=0.012)[19,25]$. Additionally, patients in the HTG pancreatitis cohort had lower adjusted odds of severity indices, which included sepsis, septic shock, NSTEMI, and need for transfusion requirements. This finding differs from current literature which has found either no statistically significant difference between HTG-induced pancreatitis and other causes of pancreatitis, including gallbladder disease, or higher disease severity in patients with HTG-induced pancreatitis $[4,13,16,19,20,25]$.

Compared to studies that tested this data in specific populations, or using smaller sample sizes, our study conducted a large nationwide analysis of data which increases the power of the study and renders it less prone to selection bias. In agreement with our study finding, another large-scale population-based study investigating HTG-induced pancreatitis found lower mortality in patients with acute pancreatitis and HTG. These findings lend credibility to the possibility that the commonly accepted idea of HTG-induced pancreatitis being associated with increased disease severity when compared to other causes of pancreatitis is incorrect [26].

We postulate that the comparatively reduced morbidity and mortality seen in HTG pancreatitis compared to gallstone-induced pancreatitis is a consequence of the increased utilization of invasive management for patients with biliary pancreatitis. Due to the high recurrence rate of biliary pancreatitis that occurs when patients do not undergo definitive management, strategies to manage gallstone pancreatitis have shifted towards performing necessary interventions (endoscopic retrograde cholangiopancreatography and cholecystectomy) before patient discharge [27,28]. Although necessary, these procedures increase the risk of complications including sepsis, septic shock, NSTEMI, and increased blood transfusion requirements. Compared to HTG-induced pancreatitis which typically is managed conservatively, the need for invasive procedures in the management of gallstone pancreatitis theoretically should be associated with higher hospitalization charges and increased length of hospitalizations. This postulation was supported by our finding of shorter hospital stay and lower total hospital charges in patients with HTG-induced pancreatitis compared to gallstone-induced disease $[2,6,17,29]$. 
with HTG-induced pancreatitis, our cohort of patients with HTG-induced pancreatitis was older than the non-HTG cohort (56.7 vs 50.8 years, p<0.001) [14]. Our study also demonstrated that patients with HTGinduced pancreatitis had a higher proportion of comorbid diabetes, obesity, and hypothyroidism when compared to the non-HTG cohort.

Taking the results of this study into consideration, patients with gallstone pancreatitis possibly have more severe pancreatitis when compared to HTG-induced disease, and thus warrant close monitoring for the development of sepsis, shock, NSTEMI, and transfusion dependence, specifically when postinvasive intervention.

The limitations of this study are as follows. Despite being amongst the three leading causes of acute pancreatitis, there is no dedicated ICD-10 code for HTG-induced pancreatitis, making data query a challenge. This obstacle was overcome by excluding other known causes of acute pancreatitis from the study population and then extracting patients with diagnoses of HTG. Secondly, the limitations of the NIS database did not allow us to stratify levels of HTG and measure the possible effect it may have on outcomes. Given the fact that the level of HTG has not been shown to correlate with disease severity in current literature, our concerns were partially remedied. Lastly, the retrospective nature of this study establishes associations but does not imply causality.

Despite these limitations, our large sample size, scientific questioning, and analysis technique help contribute new information to a largely understudied topic of outcomes of HTG-induced pancreatitis when compared to gallstone pancreatitis. Through this study, we hope to increase clinician suspicion for pancreatitis-related complications and look to encourage more large-scale investigations.

\section{Conclusions}

Large-scale studies that comparatively investigate morbidity and mortality outcomes in HTG and gallstoneinduced pancreatitis are infrequent. While some studies have found no difference in outcomes, other smaller-scale studies suggest increased disease severity in HTG-induced pancreatitis. To the best of our knowledge, this study represents one of few large-scale, nationwide studies to evaluate outcomes of HTGinduced pancreatitis compared to biliary-induced pancreatitis. Results from our study suggest that patients with HTG pancreatitis possibly have better inpatient outcomes including mortality compared to those with biliary pancreatitis. Taking the results of this study into consideration, patients with gallstone pancreatitis warrant close monitoring for the development of sepsis, shock, NSTEMI, and transfusion dependence, especially after invasive procedures.

\section{Additional Information \\ Disclosures}

Human subjects: All authors have confirmed that this study did not involve human participants or tissue. Animal subjects: All authors have confirmed that this study did not involve animal subjects or tissue. Conflicts of interest: In compliance with the ICMJE uniform disclosure form, all authors declare the following: Payment/services info: All authors have declared that no financial support was received from any organization for the submitted work. Financial relationships: All authors have declared that they have no financial relationships at present or within the previous three years with any organizations that might have an interest in the submitted work. Other relationships: All authors have declared that there are no other relationships or activities that could appear to have influenced the submitted work.

\section{References}

1. Fortson MR, Freedman SN, Webster PD III: Clinical assessment of hyperlipidemic pancreatitis . Am J Gastroenterol. 1995, 90:2134-2139.

2. Toskes PP: Hyperlipidemic pancreatitis. Gastroenterol Clin North Am. 1990, 19:783-791.

3. Rawla P, Sunkara T, Thandra KC, Gaduputi V: Hypertriglyceridemia-induced pancreatitis: updated review of current treatment and preventive strategies. Clin J Gastroenterol. 2018, 11:441-448. 10.1007/s12328-0180881-1

4. Anderson F, Thomson SR, Clarke DL, Buccimazza I: Dyslipidaemic pancreatitis clinical assessment and analysis of disease severity and outcomes. Pancreatology. 2009, 9:252-257. 10.1159/000212091

5. Ewald N: Hypertriglyceridemia-induced acute pancreatitis. Clin Lipidol. 2013, 8:587-594.

6. Chang CC, Hsieh YY, Tsai HD, Yang TC, Yeh LS, Hsu TY: Acute pancreatitis in pregnancy. Zhonghua Yi Xue Za Zhi (Taipei). 1998, 61:85-92.

7. Ames R: Hyperlipidemia of diuretic therapy . Arch Mal Coeur Vaiss. 1998, 91:23-27.

8. Bagdade JD, Yee E, Albers J, Pykalisto OJ: Glucocorticoids and triglyceride transport: effects on triglyceride secretion rates, lipoprotein lipase, and plasma lipoproteins in the rat. Metabolism. 1976, 25:533-542. 10.1016/0026-0495(76)90007-x

9. Bjornstad P, Eckel RH: Pathogenesis of lipid disorders in insulin resistance: a brief review . Curr Diab Rep. 2018, 18:127. 10.1007/s11892-018-1101-6

10. Ghio A, Bertolotto A, Resi V, Volpe L, Di Cianni G: Triglyceride metabolism in pregnancy. Adv Clin Chem. 2011, 55:133-153. 10.1016/b978-0-12-387042-1.00007-1

11. Hermans MP, Valensi P: Elevated triglycerides and low high-density lipoprotein cholesterol level as marker 
of very high risk in type 2 diabetes. Curr Opin Endocrinol Diabetes Obes. 2018, 25:118-129. 10.1097/med.0000000000000398

12. Laws A, Stefanick ML, Reaven GM: Insulin resistance and hypertriglyceridemia in nondiabetic relatives of patients with noninsulin-dependent diabetes mellitus. J Clin Endocrinol Metab. 1989, 69:343-347. 10.1210/jcem-69-2-343

13. Garg R, Rustagi T: Management of hypertriglyceridemia induced acute pancreatitis . BioMed Res Int. 2018, 2018:4721357. 10.1155/2018/4721357

14. Sekimoto M, Takada T, Kawarada Y, et al.: JPN Guidelines for the management of acute pancreatitis: epidemiology, etiology, natural history, and outcome predictors in acute pancreatitis. J Hepatobiliary Pancreat Surg. 2006, 13:10-24. 10.1007/s00534-005-1047-3

15. Durrington PN, Twentyman OP, Braganza JM, Miller JP: Hypertriglyceridaemia and abnormalities of triglyceride catabolism persisting after pancreatitis. Int J Pancreatol. 1986, 1:195-203.

16. Lloret Linares C, Pelletier AL, Czernichow S, et al.: Acute pancreatitis in a cohort of 129 patients referred for severe hypertriglyceridemia. Pancreas. 2008, 37:13-22. 10.1097/MPA.0b013e31816074a1

17. Scherer J, Singh VP, Pitchumoni CS, Yadav D: Issues in hypertriglyceridemic pancreatitis: an update . J Clin Gastroenterol. 2014, 48:195-203. 10.1097/01.mcg.0000436438.60145.5a

18. Valdivielso P, Ramírez-Bueno A, Ewald N: Current knowledge of hypertriglyceridemic pancreatitis . Eur J Intern Med. 2014, 25:689-694. 10.1016/j.ejim.2014.08.008

19. Deng L-H, Xue P, Xia Q, Yang X-N, Wan M-H: Effect of admission hypertriglyceridemia on the episodes of severe acute pancreatitis. World J Gastroenterol. 2008, 14:4558-4561. 10.3748/wjg.14.4558

20. Kimura T, Toung JK, Margolis S, Permutt S, Cameron JL: Respiratory failure in acute pancreatitis: a possible role for triglycerides. Ann Surg. 1979, 189:509-514.

21. Warshaw AL, Lesser PB, Rie M, Cullen DJ: The pathogenesis of pulmonary edema in acute pancreatitis . Ann Surg. 1975, 182:505-510. 10.1097/00000658-197510000-00016

22. Balachandra S, Virlos IT, King NK, Siriwardana HP, France MW, Siriwardena AK: Hyperlipidaemia and outcome in acute pancreatitis. Int J Clin Pract. 2006, 60:156-159. 10.1111/j.1742-1241.2005.00645.x

23. Healthcare Cost and Utilization Project. Introduction to the HCUP National Inpatient Sample (NIS) . (2017). Accessed: September 9, 2020: https://www.hcup-us.ahrq.gov/db/nation/nis/NIS_Introduction_2017.jsp.

24. Ojemolon PE, Shaka H, Edigin E, et al.: Impact of diabetes mellitus on outcomes of patients with knee osteoarthritis who underwent knee arthroplasty: an analysis of the Nationwide Inpatient Sample. Cureus. 2020, 12:e8902. 10.7759/cureus.8902

25. Navarro S, Cubiella J, Feu F, Zambon D, Fernandez-Cruz L, Ros E: Hypertriglyceridemic acute pancreatitis. Is its clinical course different from lithiasic acute pancreatitis? (Article in Spanish). Med Clin. 2004, 123:567570. 10.1016/s0025-7753(04)74599-6

26. Simons-Linares CR, Jang S, Sanaka M, et al.: The triad of diabetes ketoacidosis, hypertriglyceridemia and acute pancreatitis. How does it affect mortality and morbidity?: A 10-year analysis of the National Inpatient Sample. Medicine. 2019, 98:e14378. 10.1097/md.0000000000014378

27. Larson SD, Nealon WH, Evers BM: Management of gallstone pancreatitis. Adv Surg. 2006, 40:265-284. 10.1016/j.yasu.2006.06.005

28. Vitale GC: Early management of acute gallstone pancreatitis . Ann Surg. 2007, 245:18-19. 10.1097/01.sla.0000250967.32581.c9

29. Stefanutti C, Labbadia G, Morozzi C: Severe hypertriglyceridemia-related acute pancreatitis. Ther Apher Dial. 2013, 17:130-137. 10.1111/1744-9987.12008 